(2) Open Access Full Text Article

\title{
Use of letrozole and clomiphene citrate combined with gonadotropins in clomiphene-resistant infertile women with polycystic ovary syndrome:
} a prospective study

\author{
This article was published in the following Dove Press journal: \\ Drug Design, Development and Therapy \\ II November 2015 \\ Number of times this article has been viewed
}

\section{Wenyan $\mathrm{Xi}^{1}$ \\ Shankun Liu ${ }^{2}$ \\ Hui Mao' \\ Yongkang Yang' \\ Xiang Xue' \\ Xiaoning Lu'}

'The Second Affiliated Hospital of Xi'an Jiaotong University, Xi'an City, Shaanxi, ${ }^{2}$ Taian City Central Hospital, Shandong, Taian, People's Republic of China
Correspondence: Xiang Xue The Second Affiliated Hospital of Xi'an Jiaotong University, No 157, Xiwu Road, Xi'an City, Shaanxi Province 710004,

People's Republic of China

Tel +86 I3072906155

Email xwyanzi@126.com
Background: Gonadotropin has been used to stimulate ovulation in clomiphene-resistant infertile women with polycystic ovary syndrome (PCOS), but it is associated with overstimulated cycles with the development of many follicles. The aim of the study was to evaluate the effectiveness and efficacy of letrozole and clomiphene citrate (CC) combined with human menopausal gonadotropin (HMG) in CC-resistant infertile women with PCOS.

Methods: Ninety-four women received the letrozole $+\mathrm{HMG}, 90$ women received CC $+\mathrm{HMG}$, and 71 women received HMG only. All women received one treatment regimen in one treatment cycle. All patients were given HMG 75 IU on alternate days daily starting on day 3 or day 7 until the day of administration of human chorionic gonadotropin.

Results: The rate of monofollicular development was $80.2 \%$ in the letrozole + HMG group, $65.3 \%$ in the CC + HMG group, and $54.7 \%$ in the HMG-only group $(P<0.05$ for letrozole + HMG vs the other two groups). The number of developing follicles ( $\geq 14 \mathrm{~mm}$ follicles) and the cycle cancellation rate due to ovarian hyperresponse were the lowest in the letrozole + HMG group, but the difference was not significant. The ovulation and pregnancy rate were similar among the three protocols. The HMG dose needed and the mean duration of treatment were significantly lower in the letrozole $+\mathrm{HMG}$ and CC $+\mathrm{HMG}$ groups compared with the HMG-only group.

Conclusion: Letrozole in combination with $\mathrm{HMG}$ is an effective protocol for reducing the risks of hyperstimulation for ovarian induction in CC-resistant women with PCOS. This combination may be more appropriate in patients who are particularly sensitive to gonadotropin.

Keywords: letrozole, clomiphene citrate, human menopausal gonadotropin, combination therapy, polycystic ovary syndrome

\section{Introduction}

Polycystic ovary syndrome (PCOS) is the most common endocrine disorder in women of reproductive age, with an estimated prevalence of $5 \%-10 \%$ of the general population, and by far the most common cause of anovulatory infertility. ${ }^{1}$ For many years, clomiphene citrate (CC) has been the standard treatment for ovulation induction for these patients. It is not, however, equally successful in all situations. Approximately $25 \%$ of women with PCOS will not respond at all to $\mathrm{CC}$ and are considered to be "clomiphene resistant". ${ }^{2}$ In addition, CC may have an antiestrogenic effect on endometrial development and cervical mucus production, which have been implicated in a low pregnancy rate despite a relatively high ovulation rate with the use of this drug. 3,4 
Alternative treatments to $\mathrm{CC}$ with aromatase-inhibiting drugs such as letrozole have attracted attention in the past few years. ${ }^{2}$ Letrozole is a potent, reversible, and highly selective aromatase inhibitor that prevents androgen-to-estrogen conversion. It works by suppressing estrogen production, decreasing the negative feedback of estrogens in the hypothalamus, and subsequently increasing the circulating concentration of follicle stimulating hormone (FSH), and has been used to induce ovulation. Letrozole is considered ideal for ovulation induction, as it does not deplete estrogen receptors in central and peripheral target tissues, it may have no negative impact on endometrium and cervical mucus, and it typically results in mono-ovulation. ${ }^{5,6}$

Gonadotropin preparations, either urinary or recombinant FSH, have been used to stimulate ovulation in women who fail to ovulate or get pregnant with CC. ${ }^{7,8}$ Chronic low-dose gonadotropin have been widely used over the last decade in a step-up regimen, with initial daily doses of $75 \mathrm{IU}$ for 2 weeks and gradually increased by $37.5 \mathrm{IU}$ in an attempt to slowly and prudently surpass the individual FSH threshold for follicular recruitment. ${ }^{9-11}$ Low-dose step-up gonadotropin therapy, however, may still lead to overstimulated cycles with the development of many follicles, resulting in cycle cancellation, severe ovarian hyperstimulation syndrome (OHSS), or multiple pregnancies in patients with PCOS. ${ }^{12}$

Recombinant FSH ( $\mathrm{rFSH}$ ) used in combination with $\mathrm{CC}$ for CC-resistant PCOS was proven to be more effective in improving the ovulation rate than $\mathrm{rFSH}$ alone in a more recent study. ${ }^{13}$ However, the results have been doubted for the inconsistent data on the ovulation rate. ${ }^{14}$ In addition, $\mathrm{CC}$ use is associated with lower pregnancy rates because of its peripheral antiestrogenic effects. Because of the clear benefits of letrozole mentioned above, we proposed the possibility of using it in combination with gonadotropins for ovarian induction for an efficacious approach in women with PCOS. Although previous studies have shown that co-treatment with the letrozole significantly reduced the FSH dose required during controlled ovarian stimulation $(\mathrm{COH})$ in women with unexplained infertility, ${ }^{15,16}$ there are no studies concerning this combination therapy for patients with anovulatory PCOS.

In our department, human menopausal gonadotropins (HMGs), either alone or combined with CC or letrozole, are the options for ovarian induction in CC-resistant women with PCOS and performed depending on the physician's preference. As yet, there is no evidence that one regimen offers advantages over the other in terms of efficacy. The lack of data prompted us to conduct this prospective study to test the hypothesis that the use of letrozole or $\mathrm{CC}$, in conjunction with $\mathrm{HMG}$, would be an effective ovulation inducing agent in women with CC-resistant PCOS when compared with HMG alone.

\section{Patients and methods \\ Patients}

The study was approved by the Ethics Committee of The Second Affiliated Hospital of Xi'an Jiaotong University. All participants provided their informed consent before their involvement in this study. A total of 255 women with CC-resistant PCOS were included in the present study at the Department of Obstetrics and Gynecology. Data were collected prospectively between February 2012 and June 2014. The diagnosis of PCOS was based on the Rotterdam criteria, in which at least two of the following three criteria were met: oligomenorrhea or amenorrhea, clinical hyperandrogenism and/or hyperandrogenemia, and polycystic ovaries. ${ }^{17}$ The luteinizing hormone (LH)/FSH ratio was not taken into account since there is some controversy over its reliability as a diagnostic criterion for PCOS. All patients presented with oligo/amenorrhea or anovulatory cycles for at least 2 years. $\mathrm{CC}$ resistance was defined as failure to ovulate after three cycles with CC (100 mg/day for 5 days).

The inclusion criteria included the following: CCresistant PCOS women who were 35 years old or younger; a normal hysterosalpingogram or laparoscopy; no recorded history of pelvic surgery and/or pelvic inflammatory disease; and no history of treatment by exogenous gonadotropin. Male partners all had a normal semen analysis, according to the 2010 World Health Organization criteria, within the preceding 6 months. Exclusion criteria were as follows: the presence of any infertility factors other than anovulatory PCOS, such as hyperprolactinemia, hypothalamic amenorrhea, premature ovarian failure, and ovarian tumor.

\section{Study design}

This was a nonrandomized, prospective study. The three standard protocols that are usually applied for ovarian induction in CC-resistant infertile women with PCOS in our study center are the use of HMG either alone or in conjunction with letrozole or CC. The addition of CC or letrozole was usually decided according to the preference of the primary treating physician at the units. The treatment protocol was decided during a consultation visit prior to starting the treatment cycle.

Two hundred and fifty-five women were identified as CC-resistant and underwent ovarian induction with 
letrozole + HMG, CC + HMG, or HMG alone. Out of these, 94 patients received letrozole $+\mathrm{HMG}$, and 90 patients received $\mathrm{CC}+\mathrm{HMG}$, constituting two study groups. Letrozole $2.5 \mathrm{mg}$ /day or CC $50 \mathrm{mg}$ /day was administered from day 3 to day 7 of a spontaneous or progestogen-induced withdrawal of bleeding, plus HMG 75 IU on alternate days daily starting from day 7 and maintained for up to 10 days unless follicle maturity was reached so that human chorionic gonadotropin (hCG) could be administered. Seventy-one patients received HMG injection, constituting the control group for the first two study groups. HMG was administered in a step-up protocol starting with 75 IU on alternate days daily from cycle day 3 and maintained for up to 14 days. All patients received human menopausal gonadotropin (HMG, Li Zhu Pharmacy, People's Republic of China). We included in the trial only women undergoing their first treatment cycle using this protocol.

All patients were monitored by both transvaginal ultrasound measurement of the mean follicular diameter as well as serial assays of estradiol and LH levels on day 11 and every 1-3 days thereafter. If no response was detected (ie, no follicle $>10 \mathrm{~mm}$ in diameter) after 14 days of therapy (cycle day 17), the HMG dose was increased to $75 \mathrm{IU}$ once daily for another week. Increments from then on were by $37.5 \mathrm{IU}$ per week. The increase was continued until ovarian activity was seen on ultrasound; then, the current dose was continued until follicles reached a diameter of $\geq 18 \mathrm{~mm}$. hCG (10,000 IU) was given as a single intramuscular injection to trigger ovulation. Patients were advised for intercourse 24-36 hours after hCG injection. hCG administration was discontinued when more than three follicles reached a diameter of $\geq 18 \mathrm{~mm}$ or seven follicles were $\geq 12 \mathrm{~mm}$. Treatment with HMG was withheld if there was no ovarian response on day 28-35 of the menstrual cycle or in case of premature increase in LH. Midluteal serum progesterone was measured to confirm ovulation ( $>8 \mathrm{ng} / \mathrm{mL}$, as measured by the local laboratory). Ovulation could also be confirmed by ultrasound. Clinical pregnancy was defined as the presence of a gestational sac with cardiac activity, as detected by transvaginal ultrasound after 35 days of ovulation. A multiple pregnancy was defined as one in which there was more than one fetus.

The end points were to evaluate 1) the ovulation rate and the percentage of complete cycles with monofollicular development (one follicle $\geq 18 \mathrm{~mm}$ at the time of hCG administration), 2) the mean number of follicles $\geq 14 \mathrm{~mm}$ at the time of hCG administration in complete cycles, 3 ) the percentage of canceled cycles due to ovarian hyperresponse or no response, 4) the duration of HMG stimulation and the total HMG consumption per complete cycle, and 5) the pregnancy rate and the multiple pregnancy rate.

\section{Assessments}

Screening tests included routine measurement of early follicular phase FSH, LH, estradiol $\left(\mathrm{E}_{2}\right)$, and testosterone levels on cycle day 3 before the commencement of treatment. In the same morning of the blood tests, a transvaginal ultrasound examination was performed to assess the antral follicles count (AFC) and exclude any pelvic pathology. Follicles with diameters $\leq 10 \mathrm{~mm}$ were included in the AFC tally (ie, 2-10 mm). AFC were subgraded as 12-19, 20-29, 30-39, and $\geq 40$ small antral follicles in polycystic ovary cases.

\section{Statistical analysis}

Data are presented as mean $\pm \mathrm{SD}$. Data analysis was performed by using Statistical Package for Social Sciences (SPSS) for Windows, Version 13.0 (Chicago, IL, USA). The following statistical tests were used to analyze the various data among the three groups (two study groups and one control group). We used one-way analysis of variance (ANOVA) or the Student's $t$-test to compare the means, Kruskal-Wallis test to compare medians, and the $\chi^{2}$-test to compare proportions; $P<0.05$ was considered statistically significant.

A logistic regression analysis was performed to determine the predictors for monofollicle development. The tested independent variables included age, body mass index (BMI), menstrual patterns, T, LH/FSH ratio, AFC, and homeostasis model assessment insulin resistance index (HOMA-IR). In addition, a linear regression analysis was performed to check for the influence of several parameters on the duration of treatment. The tested parameters included age, BMI, menstrual patterns, T, LH/FSH ratio, AFC, and HOMA-IR.

\section{Results}

Population characteristics among the three groups are summarized in Table 1. No statistical difference could be detected for mean age, BMI, or menstrual patterns (amenorrhea or oligomenorrhea) among the three groups of patients. All groups had comparable proportions of primary or secondary infertility, and also the duration of infertility was not significantly different among the three groups. There was also no significant difference with regard to biochemical parameters such as FSH, LH, testosterone plasma levels, LH/FSH ratio, HOMA-IR, and AFC within the three groups. All patients studied had morphological features of PCOS on transvaginal sonographies. 
Table I Patient characteristics in three treated groups

\begin{tabular}{|c|c|c|c|}
\hline Variable & $\begin{array}{l}\text { LE + HMG } \\
(n=94)\end{array}$ & $\begin{array}{l}\text { CC + HMG } \\
(n=90)\end{array}$ & $\begin{array}{l}\text { HMG } \\
(n=7 I)\end{array}$ \\
\hline Age (years) & $27.28 \pm 3.15$ & $26.79 \pm 2.61$ & $26.93 \pm 2.75$ \\
\hline BMI $\left(\mathrm{kg} / \mathrm{m}^{2}\right)$ & $23.84 \pm 2.62$ & $23.70 \pm 3.17$ & $24.49 \pm 2.85$ \\
\hline Amenorrhea, n (\%) & $27(29)$ & $28(31.1)$ & $19(26.8)$ \\
\hline Oligomenorrhea & $66(7 \mathrm{I})$ & $72(68.9)$ & $52(73.2)$ \\
\hline $\begin{array}{l}\text { Duration of infertility } \\
\text { (years) }\end{array}$ & $3.16 \pm 1.25$ & $3.2 \mathrm{I} \pm 1.47$ & $3.09 \pm 1.45$ \\
\hline Primary infertility, n (\%) & $76(81.7)$ & $72(80)$ & $54(76.1)$ \\
\hline $\mathrm{LH}$ & $8.19 \pm 3.37$ & $7.87 \pm 3.09$ & $8.03 \pm 2.75$ \\
\hline $\mathrm{FSH}$ & $6.37 \pm 1.3$ & $6.29 \pm 1.32$ & $6.34 \pm 1.43$ \\
\hline LH/FSH ratio & $1.28 \pm 0.54$ & $1.26 \pm 0.6$ & $\mid .27 \pm 0.5 \mathrm{I}$ \\
\hline Testosterone $(\mathrm{ng} / \mathrm{mL})$ & $0.52 \pm 0.17$ & $0.5 I \pm 0.19$ & $0.54 \pm 0.21$ \\
\hline HOMA-IR & $3.18 \pm 1.98$ & $3.26 \pm I .5 \mid$ & $3.26 \pm 1.83$ \\
\hline \multicolumn{4}{|l|}{ AFC, n (\%) } \\
\hline $12-19$ follicles & $52(55.3)$ & $48(53.3)$ & $29(40.8)$ \\
\hline 20-29 follicles & $31(33)$ & $35(38.9)$ & $38(53.5)$ \\
\hline 30-39 follicles & $8(8.5)$ & $6(6.7)$ & $3(4.2)$ \\
\hline$\geq 40$ follicles & $3(3.2)$ & $\mathrm{I}(\mathrm{I} . \mathrm{I})$ & $\mathrm{I}(1.4)$ \\
\hline
\end{tabular}

Notes: Data are expressed as mean \pm standard deviation or $\mathrm{n}(\%)$. All P-values were not statistically significant.

Abbreviations: CC, clomiphene citrate; BMI, body mass index; FSH, follicle stimulating hormone; HMG, human menopausal gonadotropin; LE, letrozole; $\mathrm{LH}$, luteinizing hormone; HOMA-IR, homeostasis model assessment for insulin resistance index; $A F C$, antral follicle count.

As shown in Tables 2-4, a total of 206 patients completed cycles. Twenty-six patients did not receive HCG due to the risk of ovarian hyperstimulation. Twenty-three patients discontinued HMG treatment because of no response and premature increase in LH. The total amount of HMG used in the treatment cycles and the mean duration of treatment were significantly lower $(P<0.05)$ in both study groups (letrozole + HMG group and $\mathrm{CC}+\mathrm{HMG}$ group) when compared with the control group (HMG only) but not between the two study groups when compared with each other. The ovulation rate was $86.2 \%$ in the letrozole $+\mathrm{HMG}$ group, $80 \%$ in the $\mathrm{CC}+\mathrm{HMG}$ group, and $74.6 \%$ in the HMG-only group; this difference was not statistically significant. The rate of monofollicular development was $80.2 \%$ in the letrozole + HMG group, $65.3 \%$ in the CC + HMG group, and $54.7 \%$ in the HMG-only group, which was significantly higher in the letrozole $+\mathrm{HMG}$ group when compared with the other two groups $(P<0.05)$. The number of developing follicles ( $\geq 14 \mathrm{~mm}$ follicles) and cycle cancellation rate due to ovarian hyperresponse were lowest in the letrozole + HMG groups, but the difference was not significant. When logistic regression analysis was performed, no parameters predicted monofollicle development. Similarly, no parameters were significantly associated with the duration of stimulation (data not shown). Estradiol level on the day of hCG administration was significantly lower in subjects receiving letrozole + HMG than those who received HMG alone (537.9 \pm 197.1 vs $639.1 \pm 233.3 \mathrm{pg} / \mathrm{mL}, P<0.05$ ), but not for the CC + HMG group compared with the other two groups. No statistically significant differences were found for the endometrial thickness on the day of hCG administration and for the cancellation rate due to no response.

In addition, the respective clinical pregnancy rates per noncanceled cycles were similar among the three protocols $(24.7 \%$ vs $23.6 \%$ vs $22.6 \%$ ). One twin pregnancy occurred in the $\mathrm{CC}+$ HMG group and two in the HMG-only group. Five patients, two each from the letrozole $+\mathrm{HMG}$ and $\mathrm{CC}+\mathrm{HMG}$ groups and one from the HMG-only group, had a first trimester loss. The number was too small to make any statistical inference. There were no ovarian OHSS, triplet pregnancy, or ectopic pregnancy documented in any of the three groups.

\section{Discussion}

We have shown in this study that co-treatment with the letrozole significantly reduced the duration of stimulation and total

Table 2 Clinical results in all treatment cycles: letrozole + HMG group vs HMG group

\begin{tabular}{|c|c|c|c|}
\hline Variable & Letrozole + HMG $(n=94)$ & HMG (n=7I) & $P$-value \\
\hline Complete cycle & 81 & 53 & \\
\hline Duration of treatment (days) & $17.52 \pm 3.50$ & $19.24 \pm 3.25$ & $<0.01$ \\
\hline Total amount of HMG (IU) & $7 \mid 4.8 \pm 265.5$ & $991.5 \pm 242.1$ & $<0.001$ \\
\hline $\mathrm{E}_{2}$ concentration on day of $\mathrm{hCG}(\mathrm{pg} / \mathrm{mL})$ & $537.9 \pm 197.1$ & $639.1 \pm 233.3$ & $<0.01$ \\
\hline Endometrial thickness at hCG (mm) & $9.98 \pm 1.72$ & $10.3 \pm 1.89$ & NS \\
\hline Number of follicles $\geq 14 \mathrm{~mm} /$ complete cycle & $1.75 \pm 0.89$ & $2.04 \pm 0.91$ & NS \\
\hline Monofollicular cycles/complete cycle & $65 / 81(80.2)$ & $29 / 53(54.7)$ & $<0.01$ \\
\hline Canceled cycles due to ovarian hyperresponse & $5(5.4)$ & $10(14.1)$ & NS \\
\hline Canceled cycles due to no ovarian response & $5(5.4)$ & $7(9.9)$ & NS \\
\hline Canceled cycles due to premature LH increase & $3(3.2)$ & I ( $(1.4)$ & NS \\
\hline Ovulation/started cycle (\%) & $81 / 94(86.2)$ & $53 / 7 \mid(74.6)$ & NS \\
\hline Pregnancies/complete cycle (\%) & 20/8I (24.7) & I2/53 (22.6) & NS \\
\hline
\end{tabular}

Notes: Data are expressed as mean \pm standard deviation or $n(\%) . P<0.05$ was considered statistically significant.

Abbreviations: $E_{2}$, estradiol; hCG, human chorionic gonadotropin; HMG, human menopausal gonadotropin; LH, luteinizing hormone; NS, not significant. 
Table 3 Clinical results in all treatment cycles: CC + HMG group vs HMG group

\begin{tabular}{|c|c|c|c|}
\hline Variable & CC + HMG $n=90$ & HMG $n=7$ I & $P$-value \\
\hline Complete cycle & 72 & 53 & \\
\hline Duration of treatment (days) & $17.75 \pm 3.87$ & $19.24 \pm 3.25$ & $<0.05$ \\
\hline Total amount of HMG (IU) & $731.3 \pm 290.3$ & $991.5 \pm 242.1$ & $<0.001$ \\
\hline$E_{2}$ concentration on day of $h C G(p g / m L)$ & $586.4 \pm 259.2$ & $639.1 \pm 233.3$ & NS \\
\hline Endometrial thickness at hCG (mm) & $9.9 \pm 1.78$ & $10.3 \pm 1.89$ & NS \\
\hline Number of follicles $>14 \mathrm{~mm} /$ complete cycle & $1.88 \pm 0.9$ & $2.04 \pm 0.91$ & NS \\
\hline Monofollicular cycles/complete cycle & $47 / 72(65.3)$ & $29 / 53(54.7)$ & NS \\
\hline Canceled cycles due to ovarian hyperresponse & II (I2.2) & $10(14.1)$ & NS \\
\hline Canceled cycles due to no ovarian response & $5(5.6)$ & $7(9.9)$ & NS \\
\hline Canceled cycles due to premature $\mathrm{LH}$ increase & $2(2.2)$ & $\mathrm{I}(\mathrm{I} .4)$ & NS \\
\hline Ovulation/started cycle (\%) & $72 / 90(80)$ & $53 / 7 \mid(74.6)$ & NS \\
\hline Pregnancies/complete cycle (\%) & $17 / 72(23.6)$ & $12 / 53(22.6)$ & NS \\
\hline
\end{tabular}

Notes: Data are expressed as mean \pm standard deviation or $n(\%) . P<0.05$ was considered statistically significant.

Abbreviations: CC, clomiphene citrate; $\mathrm{E}_{2}$, estradiol; hCG, human chorionic gonadotropin; HMG, human menopausal gonadotropin; LH, luteinizing hormone; NS, not significant.

HMG dose needed for stimulation, similar to co-treatment with CC. Moreover, the rate of monofollicular development was significantly higher when letrozole was added to HMG. The regimen of letrozole $+\mathrm{HMG}$ was therefore more effective and safer than $\mathrm{CC}+\mathrm{HMG}$ or HMG alone for ovulation induction in cases of $\mathrm{CC}$ resistance. These results support the concept that letrozole combined with $\mathrm{HMG}$ reduces the risk of hyperstimulation in patients who are particularly sensitive to gonadotropins. To the best of our knowledge, there are no studies evaluating the effects of letrozole co-administered during exogenous gonadotropin treatment using the low-dose step-up protocol in CC-resistant women with PCOS.

In ovulation induction, the aim should be to achieve the ovulation of a single follicle and hence to reduce the risks of OHSS and multiple pregnancies in women with PCOS. ${ }^{18-20}$ However, the problem of achieving the desired monofollicular ovulation is particularly difficult and acute due to the extreme sensitivity of the polycystic ovary to gonadotrophic stimulation. It was reported that $\sim 10 \%-20 \%$ of cycles were abandoned before completion because of ovarian hyperresponse, ${ }^{21-24}$ and the ovulation of a single dominant follicle was attained in only $\sim 50 \%$ of noncanceled cycles. ${ }^{22-24}$ In this trial, the percentage of unifollicular development was significantly higher in the letrozole $+\mathrm{HMG}$ group $(80.2 \%)$ compared to the CC + HMG (65.3\%) and HMG-only (54.7\%) groups. In addition, there was a trend toward a lower number of developing follicles and cycle cancellation rate due to ovarian hyperresponse in the letrozole + HMG group.

These results are consistent with the major advantage of letrozole in achieving the restoration of monofollicular ovulation in women with PCOS. Letrozole is completely absorbed after oral administration with a mean terminal half-life of $\sim 45$ hours. ${ }^{25}$ In addition, Letrozole does not deplete estrogen receptors, and normal negative feedback mechanisms for

Table 4 Clinical results in all treatment cycles: letrozole + HMG group vs CC + HMG group

\begin{tabular}{|c|c|c|c|}
\hline Variable & Letrozole + HMG $(n=94)$ & CC + HMG $(n=90)$ & $P$-value \\
\hline Complete cycle & 81 & 72 & \\
\hline Duration of treatment (days) & $17.52 \pm 3.50$ & $17.75 \pm 3.87$ & $<0.01$ \\
\hline Total amount of HMG (IU) & $7 \mid 4.8 \pm 265.5$ & $731.3 \pm 290.3$ & $<0.001$ \\
\hline$E_{2}$ concentration on day of $h C G(p g / m L)$ & $537.9 \pm 197.1$ & $586.4 \pm 259.2$ & $<0.01$ \\
\hline Endometrial thickness at hCG (mm) & $9.98 \pm 1.72$ & $9.9 \pm 1.78$ & NS \\
\hline Number of follicles $>14 \mathrm{~mm} /$ complete cycle & $1.75 \pm 0.89$ & $1.88 \pm 0.9$ & NS \\
\hline Monofollicular cycles/complete cycle & $65 / 81(80.2)$ & $47 / 72(65.3)$ & $<0.01$ \\
\hline Canceled cycles due to ovarian hyperresponse & $5(5.4)$ & II (I2.2) & NS \\
\hline Canceled cycles due to no ovarian response & $5(5.4)$ & $5(5.6)$ & NS \\
\hline Canceled cycles due to premature $\mathrm{LH}$ increase & $3(3.2)$ & $2(2.2)$ & NS \\
\hline Ovulation/started cycle (\%) & $8 \mathrm{I} / 94(86.2)$ & $72 / 90(80)$ & NS \\
\hline Pregnancies/complete cycle (\%) & 20/8I (24.7) & $17 / 72(23.6)$ & NS \\
\hline
\end{tabular}

Notes: Data are expressed as mean \pm standard deviation or $n$ (\%). $P<0.05$ was considered statistically significant.

Abbreviations: CC, clomiphene citrate; $E_{2}$, estradiol; hCG, human chorionic gonadotropin; HMG, human menopausal gonadotropin; LH, luteinizing hormone; NS, not significant. 
FSH in the brain remain intact. ${ }^{26,27}$ However, CC results in central estrogen receptor depletion for a long time because of its greater half-time for clearance ( 2 weeks). ${ }^{28}$ As a result, supraphysiologic levels of estrogen can occur without central suppression of FSH because the normal estrogen-receptormediated feedback mechanisms are blocked. This results in multiple follicle growth and higher multiple pregnancy rates with CC than are found in letrozole cycles. These results proved that, even in conjunction with HMG, letrozole still has the advantage of achieving monofollicle development. Thus, we suggest that for women with PCOS who are at high risk of ovarian hyperresponse, letrozole + HMG may be more appropriate as compared with other approaches for ovulation induction.

However, the result may seem paradoxical, given that there were several studies that reported equal number (or more) of leading follicles developed when letrozole was added to gonadotropin in $\mathrm{COH}$. Healey et $\mathrm{a} \mathrm{l}^{15}$ evaluated women who were superovulated with gonadotropin or a combined regimen of gonadotropin and letrozole, and found that the combined regimen developed more follicles $>14 \mathrm{~mm}$. In a prospective, nonrandomized study involving women with unexplained infertility or mild male factor, the number of follicles of $>18 \mathrm{~mm}$ were similar among women in the letrozole + FSH, CC + FSH, and FSH-only groups ${ }^{16}$ It must be stressed, however, that in these studies infertile women without PCOS was included and $\mathrm{COH}$ combined with intrauterine insemination was offered. The goal of stimulation in these women was to increase the number of available oocytes for in vivo fertilization and eventually enhance the chance of pregnancy. ${ }^{29}$ So a large dose of FSH (150 IU) was given, which resulted in multifollicular development and which is different from patients with PCOS undergoing a low-dose step-up protocol (with an initial dose of 75 IU HMG on alternate days) aimed at accomplishing single follicle development in our study.

In this trial, the ovulation rate was highest in letrozole + HMG group, but the difference was not significant. The clinical pregnancy rate per noncanceled cycles was also comparable among the three groups. It is interesting to note that there was no significant benefit of combination therapy with letrozole and HMG to improve the pregnancy rate as we expected, and we could not demonstrate that the pregnancy rate was decreased in $\mathrm{CC}+\mathrm{HMG}$ group as reported in previous studies. A prospective analysis included women with unexplained infertility and found that the pregnancy rate per cycle in the CC + FSH group (10.5\%) was significantly lower than in the letrozole + FSH group (19.1\%) and the FSH-only group (18.7\%) during $\mathrm{COH} .{ }^{16}$ Ghanem et al ${ }^{13}$ reported that the discrepancy between ovulation and conception rates in the CC + FSH arm (87\% vs $29 \%)$ was greater than in the FSH arm (31.5\% vs $28.5 \%)$. This discrepancy was attributed to the peripheral anti-estrogenic effects of CC at the level of the endometrium and cervical mucus.

We suppose the possible reason for favorable pregnancy rates with $\mathrm{CC}$ in our study could be a good endometrial thickness facilitating implantation of the fertilized ovum, because the mean endometrial thickness on the day of hCG administration was the same among the three groups. It must be emphasized that the patients in our study were suffering from CC-resistant PCOS, a particularly troublesome group to treat with gonadotropin therapy. CC-resistant patients are not resistant because they are incapable of producing FSH in response to $\mathrm{CC}$, but rather because of the higher FSH requirements of their leading follicles. In other words, these patients need more gonadotropin and longer treatment, which may result in delayed ovulation in CC-resistant PCOS patients, allowing some time for the recovery of estrogen receptors and endometrial proliferation.

For clinical trials of gonadotropin treatment in women with anovulatory infertility, an important consideration is the total dose of gonadotropin required to stimulate ovulation. The present study, the total amount of HMG required and mean duration of stimulation were significantly lower in both letrozole $+\mathrm{HMG}$ and $\mathrm{CC}+\mathrm{HMG}$ groups when compared with the HMG-only group. This finding is in agreement with the above-mentioned studies. ${ }^{15,16}$

In this study, a strict exclusion criterion for HCG administration (more than three follicles $\geq 18 \mathrm{~mm}$ or seven follicles $\geq 12 \mathrm{~mm}$ ) was used. With this precaution, no OHSS was observed, and there were 46 single pregnancies (93.9\%) and three twin pregnancies (6.1\%).

It should be mentioned that the women received $75 \mathrm{IU}$ HMG on alternate days as starting doses for ovulation induction in present study, which was different from the standard low-dose step-up regimen with initial dose of 75 IU daily. Recently, the lower starting dose was employed to reduce the risks of OHSS and multiple pregnancies in patients with PCOS. Indeed, a starting dose of recombinant FSH (50 IU) administered on alternate days successfully induced uniovulation in patients with chronic anovulation secondary to PCOS. ${ }^{30}$ Moreover, a 37.5 IU starting dose of FSH daily was found to be successful in reducing the risk of OHSS while maintaining a satisfactory pregnancy rate in women with anovulatory PCOS. ${ }^{31}$ These results gave credence to a starting dose of 75 IU on alternate days for follicular development. We really achieved a satisfactory ovulation and pregnancy 
rates in the HMG-only group, similar to those reported in studies using a 75 IU starting dose. . $3,32^{2}$

However, the mean duration of treatment needed to achieve ovulation was $\sim 19$ days with HMG alone in this study, which is longer than the reported of 14-15 days in several studies that used a standard low step-up protocol. ${ }^{23,32,33}$ Thus, the use of starting doses on alternate days may result in an increased need for monitoring, and the optimal starting dose is yet to be determined. Further studies will be necessary to verify whether a starting dose of $37.5 \mathrm{IU}$ or $50 \mathrm{IU}$ daily is more appropriate to achieve better results, especially in a chronic, low-dose step-up protocol when combined with letrozole.

A significant limitation of this trial is the nonrandomized design of the study. However, because no similar studies have been reported earlier, we believe that the present observation from the large number of CC-resistant PCOS patients was important to precede any definitive randomized studies in order to test for the feasibility of the idea of using letrozole to improve monofollicular ovulation and reduce the risk of OHSS. We believe the positive results of the present trial should encourage us and others to proceed with more definitive prospective randomized clinical trials to investigate our findings.

\section{Conclusion}

In conclusion, we found letrozole co-treatment with HMG not only reduced the duration of stimulation and total HMG dose needed for stimulation but also achieved a higher incidence of monofollicular growth, demonstrating that this protocol is effective and safe for ovarian induction. According to the results of this study, it can be proposed that in patients with PCOS resistant to clomiphene, a combination of letrozole and HMG can be administered before using a more aggressive treatment that may have severe complications (such as laparoscopic ovarian diathermy or in vitro fertilization). Moreover, this combination therapy for ovulation induction may have the advantage of reducing the risks of hyperstimulation and therefore may be more appropriate in highly responsive women with PCOS.

\section{Acknowledgments}

We would like to express our gratitude to Shankun Liu for help in statistical analysis and Hui Mao for her invaluable help in the collection and storage of data. This study was supported by The Second Affiliated Hospital of Xi'an Jiaotong University.

\section{Disclosure}

The authors report no conflicts of interest in this work.

\section{References}

1. Norman RJ, Dewailly D, Legro RS, Hickey TE. Polycystic ovary syndrome. Lancet. 2007;370:685-697.

2. Mitwally MF, Casper RF. Use of an aromatase inhibitor for induction of ovulation in patients with an inadequate response to clomiphene citrate. Fertil Steril. 2001;75:305-309.

3. Gonen Y, Casper RF. Sonographic determination of a possible adverse effect of clomiphene citrate on endometrial growth. Hum Reprod. 1990; 5:670-674.

4. Randall JM, Templeton A. Cervical mucus score and in vitro sperm mucus interaction in spontaneous and clomiphene citrate cycles. Fertil Steril. 1991;56:465-468.

5. Mitwally MF, Casper RF. Aromatase inhibitors in ovulation induction. Semin Reprod Med. 2004;22:61-78.

6. Casper RF, Mohamed FM. Historical perspective of aromatase inhibitors for ovulation induction. Fertil Steril. 2012;98:1352-1355.

7. The Thessaloniki ESHRE/ASRM-Sponsored PCOS Consensus Workshop Group. Consensus on infertility treatment related to polycystic ovary syndrome. Hum Reprod. 2008;23:462-477.

8. Wang CF, Gemzell C. The use of human gonadotropins for the induction of ovulation in women with polycystic ovarian disease. Fertil Steril. 1980;33:479-486.

9. Hamilton-Fairley D, Kiddy D, Watson H, Sagle M, Franks S. Low-dose gonadotrophin therapy for induction of ovulation in 100 women with polycystic ovarian syndrome. Hum Reprod. 1991;6:1095-1099.

10. Franks S, Gilling-Smith C. Advances in induction of ovulation. Curr Opin Obstet Gynecol. 1994;6:136-140.

11. van Santbrink EJ, Fauser BC. Urinary follicle-stimulating hormone for normogonadotropic clomiphene-resistant anovulatory infertility: prospective, randomized comparison between low dose step-up and step-down dose regimens. J Clin Endocrinol Metab. 1997;82: 3597-3602.

12. Herman A, Ron-El R, Golan A, Soffer Y, Bukovsky I, Caspi E. Overstimulated cycles under low-dose gonadotrophins in patients with polycystic ovary syndrome: characterization and management. Hum Reprod. 1993;8:30-34.

13. Ghanem ME, Elboghdady LA, Hassan M, et al. Clomiphene citrate co-treatment with low dose urinary FSH versus urinary FSH for clomiphene resistant PCOS: randomized controlled trial. J Assist Reprod Genet. 2013;30:1477-1485.

14. Papathanasiou A. Comment on: "Clomiphene citrate co-treatment with low dose urinary FSH versus urinary FSH for clomiphene resistant PCOS: randomized controlled trial." by Ghanem et al. J Assist Reprod Genet. 2013;30:1525.

15. Healey S, Tan SL, Tulandi T, Biljan MM. Effects of letrozole on superovulation with gonadotropins in women undergoing intrauterine insemination. Fertil Steril. 2003;80:1325-1329.

16. Mitwally MF, Casper RF. Aromatase inhibition reduces gonadotrophin dose required for controlled ovarian stimulation in women with unexplained infertility. Hum Reprod. 2003;18:1588-1597.

17. Rotterdam ESHRE/ASRM-Sponsored PCOS Consensus Workshop Group. Revised 2003 consensus on diagnostic criteria and long-term healthy risks related to polycystic ovary syndrome. Fertil Steril. 2004;82:1193-1197.

18. Fauser BC, Van Huesden AM. Manipulation of human ovarian function: physiological concepts and clinical consequences. Endrocr Rev. 1997; 18:71-106.

19. Vegetti W, Guermandi E, Baroni E, et al. Induction of monofollicular cycles. Minerva Ginecol. 2001;53:41-48.

20. Phipps WR. Polycystic ovary syndrome and ovulation induction. Obstet Gynecol Clin North Am. 2001;28:165-182.

21. Coelingh Bennink HJ, Fauser BC, Out HJ. Recombinant folliclestimulating hormone (FSH; Puregon) is more efficient than urinary FSH (Metrodin) in women with clomiphene citrate-resistant, normogonadotropic, chronic anovulation: a prospective, multicenter, assessor-blind, randomized, clinical trial. European Puregon Collaborative Anovulation Study Group. Fertil Steril. 1998;69:19-25. 
22. Balasch J, Fábregues F, Creus M, Puerto B, Peñarrubia J, Vanrell JA. Follicular development and hormone concentrations following recombinant FSH administration for anovulation associated with polycystic ovarian syndrome: prospective, randomized comparison between low-dose step-up and modified step-down regimens. Hum Reprod. 2001;16:652-656

23. Yarali H, Bukulmez O, Gurgan T. Urinary follicle-stimulating hormone (FSH) versus recombinant FSH in clomiphene citrate-resistant, normogonadotropic, chronic anovulation: a prospective randomized study. Fertil Steril. 1999;72:276-281.

24. Hugues JN, Cedrin-Durnerin I, Avril C, Bulwa S, Herve F, Uzan M. Sequential step-up and step-down dose regimen: an alternative method for ovulation induction with follicle-stimulating hormone in polycystic ovarian syndrome. Hum Reprod. 1996;11:2581-2584.

25. Casper RF, Mitwally MF. Use of the aromatase inhibitor letrozole for ovulation induction in women with polycystic ovarian syndrome. Clin Obstet Gynecol. 2011;54:685-695.

26. Sioufi A, Gauducheau N, Pineau V, et al. Absolute bioavailability of letrozole in healthy post-menopausal women. Biopharm Drug Dispos. 1997;18:779-789.

27. Sioufi A, Sandrenan N, Godbillon J, et al. Comparative bioavailability of letrozole under fed and fasting conditions in 12 healthy subjects after a $2.5 \mathrm{mg}$ single oral administration. Biopharm Drug Dispos. 1997;18: 489-497.
28. MacDougall MJ, Tan SL, Hall V, Balen A, Mason BA, Jacobs HS. Comparison of natural with clomiphene citrate-stimulated cycles in in vitro fertilization: a prospective, randomized trial. Fertil Steril. 1994;61:1052-1057.

29. Badawy A, Elnashar A, Totongy M. Clomiphene citrate or aromatase inhibitors for superovulation in women with unexplained infertility undergoing intrauterine insemination: a prospective randomized trial. Fertil Steril. 2009;92:1355-1359.

30. Buckler HM, Robertson WR, Anderson A, Vickers M, Lambert A. Ovulation induction with low dose alternate day recombinant follicle stimulating hormone (Puregon). Hum Reprod. 1999;14:2969-2973.

31. Balasch J, Fábregues F, Creus M, Casamitjana R, Puerto B, Vanrell JA. Recombinant human follicle-stimulating homrone for ovulation induction in polycystic ovary syndrome: a prospective, randomized trial of two starting doses in a chronic low-dose step-up protocol. J Assist Reprod Genet. 2000;1:561-565.

32. Hedon B, Hugues JN, Emperaire JC, et al. A comparative prospective study of a chronic low dose versus a conventional ovulation stimulation regimen using recombinant human follicle stimulating hormone in anovulatory infertile women. Hum Reprod. 1998;13:2688-2692.

33. Christin-Maitre S, Hugues JN; Recombinant FSH Study Group. A comparative randomized multicentric study comparing the step-up versus step-down protocol in polycystic ovary syndrome. Hum Reprod. 2003;18:1626-1631.
Drug Design, Development and Therapy

\section{Publish your work in this journal}

Drug Design, Development and Therapy is an international, peerreviewed open-access journal that spans the spectrum of drug design and development through to clinical applications. Clinical outcomes, patient safety, and programs for the development and effective, safe, and sustained use of medicines are a feature of the journal, which

\section{Dovepress}

has also been accepted for indexing on PubMed Central. The manuscript management system is completely online and includes a very quick and fair peer-review system, which is all easy to use. Visit http://www.dovepress.com/testimonials.php to read real quotes from published authors.

Submit your manuscript here: http://www.dovepress.com/drug-design-development-and-therapy-journal 